\title{
Oxidized low-density lipoprotein increases the proliferation and migration of human coronary artery smooth muscle cells through the upregulation of osteopontin
}

\author{
JUN LIU, YINGANG REN, LI KANG and LIHUA ZHANG \\ Department of Geriatrics, Tangdu Hospital, The Fourth Military Medical University, Xi'an, Shaanxi 710038, P.R. China
}

Received November 5, 2013; Accepted February 21, 2014

DOI: $10.3892 /$ ijmm.2014.1681

\begin{abstract}
Smooth muscle cell (SMC) proliferation and migration are known to play a critical role in the development of atherosclerosis. Oxidized low-density lipoprotein (oxLDL) is involved in the generation of atherosclerotic lesions. Recent studies have indicated that oxLDL is a well-established risk factor for atherosclerosis that induces vascular smooth muscle cell (VSMC) proliferation and migration; however, the exact mechanisms involved have not been fully elucidated. In this study, the proliferation of human coronary artery smooth muscle cells (HCASMCs) was detected by 3-(4,5-dimethylthiazol-2-yl)-2,5-diphenyltetrazolium bromide (MTT) assay. Cell migration was determined by Transwell assay. Osteopontin (OPN), matrix metaloproteinase-9 (MMP-9) and $\alpha v \beta 3$ integrin expression were measured by mRNA and western blot analysis. OPN and MMP-9 knockdown cells were established through transfection with OPN siRNA or MMP-9 siRNA, respectively. Our results revealed that oxLDL makredly promoted HCASMC proliferation and migration in a dose-dependent manner. Further experiments demonstrated that oxLDL upregulated the expression of OPN and oxLDL. Cell proliferation and migration were markedly reduced following the knockdown of the OPN gene in the HCASMCs. We then found that treatment with oxLDL induced a concentration-dependent increase in MMP-9 mRNA and protein levels in the HCASMCs. These effects were partially abrogated by silencing OPN expression or blocking the $\alpha \mathrm{v} \beta 3$ integrin pathway. Moreover, cells treated
\end{abstract}

Correspondence to: Dr Lihua Zhang, Department of Geriatrics, Tangdu Hospital, The Fourth Military Medical University, 1 Xinsi Road, Baqiao, Xi'an, Shanxi 710038, P.R. China

E-mail: lihuazhangen@163.com

Abbreviations: SMCs, smooth muscle cells; HCASMCs, human coronary artery smooth muscle cells; ox-LDL, oxidized low-density lipoprotein; OPN, osteopontin; ECM, extracellular matrix; MMP-9, matrix metalloproteinase-9; OD, optical density; siRNA, small interfering RNA

Key words: atherosclerosis, osteopontin, matrix metaloproteinase-9, proliferation, migration with MMP-9 siRNA or $\alpha \mathrm{v} \beta 3$ antibody showed lower proliferation and migration rates. This study provides direct in vitro evidence that the exposure of HCASMCs to oxLDL induces the activation of OPN, leading to higher protein levels of MMP-9, and to an increased proliferation and migration of HCASMCs.

\section{Introduction}

Atherosclerosis is considered to be an important pathological manifestation of cardiovascular diseases and chronic inflammatory disease (1). Moreover, the disease is the major risk factor for cardiovascular disease morbidity and mortality. The development of atherosclerosis is associated with elements of the arterial wall, including $\mathrm{T}$ cells, monocyte-derived macrophages, endothelial cells and vascular smooth muscle cells (VSMCs) $(2,3)$. The accumulation of VSMCs contributes to occlusive cardiovascular pathologies, including atherosclerosis (4). VSMCs reside within the media in a quiescent state in the healthy blood vessel, whereas they migrate into the intima after injury, and their growth can result in the restriction of normal blood flow $(5,6)$. The proliferation of VSMCs plays an important role in the pathology of atherosclerosis formation, coronary heart disease, hypertension and restenosis following percutaneous transluminal coronary angioplasty (PTCA) $(7,8)$. As regards atherosclerosis, VSMC proliferation is important for plaque formation. VSMC apoptosis occurs in a number of arterial diseases, including angioplasty restenosis, aneurysm formation and atherosclerosis (9). The apoptosis of VSMCs is involved in atherosclerotic plaque rupture (10).

Osteopontin (OPN), a secreted protein, is expressed by various cell types and has chemotactic, pro-adhesive and cytokine-like properties. OPN is involved in a number of physiological and pathological events, such as bone remodeling, tumorigenesis, angiogenesis and apoptosis (11). Studies have demonstrated that OPN is a multifunctional protein that is upregulated in a wide variety of inflammatory conditions, such as autoimmune disease, fibrosis, wound healing and atherosclerosis (12). The expression of OPN is upregulated at sites with atherosclerotic plaques, particularly in those associated with macrophages and foam cells (12). OPN, a critical mediator of inflammation, plays an essential role in macrophage migration, tissue infiltration (13) and activation (14), as well as in neutrophil migration and recruitment (15). Evidence 
suggests that OPN is also expressed in smooth muscle-derived foam cells in human atherosclerotic lesions of the aorta (16). Moreover, OPN can bind to several types of integrin (such as $\alpha v \beta 3$ integrin) to promote the attachment of the cells to the extracellular matrix (ECM) and to regulate the functions of these cells through certain signaling pathways (17). It has been suggested that the inhibition of the effects of $\alpha v \beta 3$ integrin by disintegrin agents, such as S247, can weaken cancer cell migration and invasion (18). In the context of atherosclerosis, OPN is generally regarded as a pro-atherogenic molecule. An previous study indicated that OPN upregulates the expression of matrix metalloproteinase (MMP)-9 through the $\alpha v \beta 3$ integrin pathway in human chondrosarcoma cells (19).

A large number of studies have indicated that MMPs play a role in regulating the behavior of VSMCs both in vitro and in vivo (20). MMPs can regulate the migration, proliferation and survival of VSMCs. MMP-9, a member of the MMP family, can degrade basement membranes and promote neointima formation in mouse models $(21,22)$. Furthermore, there is evidence to indicate that MMP-9 knockout (KO) mice show a reduced accumulation of VSMCs in atherosclerotic plaques of brachiocephalic arteries (23). These data highlight the possibility that MMP-9 plays an essential role in the development of atherosclerosis. To the best of our knowledge, in the present study, we provide the first direct evidence that oxidized low-density lipoprotein (oxLDL) induces the proliferation and apoptosis of human VSMCs through the upregulation of OPN. We demonstrate that the expression of OPN is associated with the biological behavior of VSMCs by upregulating the levels of MMP-9 through the $\alpha v \beta 3$ integrin pathway.

\section{Materials and methods}

Ethics statement. Ethical approval was obtained from the Ethics Committee of Tangdu Hospital, the Fourth Military University, Shaanxi, China. All patients participating in the study were of Chinese origin and provided written informed consent for study sample collection and permission for research use.

Antibodies. Mouse anti-OPN, mouse anti-MMP-9, mouse anti- $\alpha v \beta 3$ integrin and mouse anti- $\beta$-actin monoclonal antibodies were purchased from Abcam (Cambridge, MA, USA). HRP-conjugated goat anti-mouse IgG was obtained from Santa Cruz Biotechnology Inc. (Santa Cruz, CA, USA).

Low-density lipoprotein (LDL) preparation and oxidation. Human LDL was purified from the fresh plasma of healthy donors by sequential centrifugation, according to a previously described method $(24,25)$. The concentrations of LDL were determined by the Lowry method (26). For the production of oxLDL, $200 \mu \mathrm{g} / \mathrm{ml} \mathrm{LDL}$ were exposed to $20 \mu \mathrm{M} \mathrm{CuSO}_{4}$ in PBS for oxidation and the oxidative reactions were terminated with $40 \mu \mathrm{M}$ butylhydroxytoluene in ethanol. Furthermore, oxLDL was dialyzed against culture medium and sterile filtered.

VSMC culture. Human coronary artery smooth muscle cells (HCASMCs) were obtained from PromoCell $\mathrm{GmbH}$ (Heidelberg, Germany). The cells were cultured at $37^{\circ} \mathrm{C}$ under a humidified atmosphere of $5 \% \mathrm{CO}_{2}$ and $95 \%$ air and were grown in SMC growth medium supplemented with $10 \%$ fetal bovine serum, $100 \mu \mathrm{g} / \mathrm{ml}$ streptomycin and $100 \mathrm{U} / \mathrm{ml}$ penicillin. HCASMCs between the third and sixth passages were used throughout this study. Cells of these passages were incubated for an additional 2 days to render them quiescent prior to the initiation of each experiment, as previously described (27). To establish the HCASMC model of atherosclerosis, oxLDL was used as previously described (7). The cells were divided into 4 groups: one group was treated with or without oxLDL (10-100 $\mu \mathrm{g} / \mathrm{ml})$; the second group was treated with or without small interfering RNA (siRNA) against OPN; the third group was treated with or without siRNA against MMP-9; and the fourth group was treated with or without $\alpha v \beta 3$ integrin antibody.

MTT proliferation assay. The proliferation of the HCASMCs was measured by 3-(4,5-dimethylthiazol-2-yl)-2,5-diphenyltetrazolium bromide (MTT) assay according to a previously described method with minor modifications (7). Briefly, approximately $1 \times 10^{4}$ cells were seeded into 96 -well plates and cultured for $24 \mathrm{~h}$. SMC growth medium was used in the normal control group, while $100 \mathrm{mg} / \mathrm{l}$ oxLDL was added to the cells in the experimental groups. The cells were then cultured at $37^{\circ} \mathrm{C}$ for $24 \mathrm{~h}$. MTT $(20 \mu \mathrm{l}, 5 \mathrm{mg} / \mathrm{ml})$ was then added to the HCASMCs for $4 \mathrm{~h}$ at $37^{\circ} \mathrm{C}$ and $200 \mu \mathrm{l}$ DMSO were added to solubilize the crystals for $25 \mathrm{~min}$ at room temperature. The optical density (OD) value was determined at $490 \mathrm{~nm}$ by a spectrophotometer (Multiskan MK3; Thermo Fisher Scientific, Waltham, MA, USA). Each experimental group had at least 5 wells and all experiments were performed at least 3 times and the average results were calculated.

Migration assay. HCASMC migration was detected by Transwell assay using Transwell Costar inserts (pore size, $8 \mu \mathrm{m}$; diameter, 6.4 mm; Corning Inc., Corning, NY, USA) according to a previously described method with minor modifications (28). Briefly, the cells were treated with siRNAs or $\alpha v \beta 3$ integrin antibody prior to the migration assays. The Transwells were coated with collagen ( $0.01 \%$; Sigma, St. Louis, MO, USA) overnight at $4^{\circ} \mathrm{C}$. The HCASMCs were grown to $80 \%$ confluence, and then detached with non-enzymatic cell dissociation buffer. Cells were resuspended in serum-free SMC medium, followed by transfer into the Transwells at a density of $1 \times 10^{4}$ cells $/ \mathrm{ml}$. The cells were placed in the upper chamber, while chemoattractants were placed in the bottom of the Transwell, and the cells were allowed to migrate for $4 \mathrm{~h}$. Non-migrated cells were removed from the upper chamber using a cotton bud, whereas the migrated cells were stained with the Reastain Quick-Diff kit (Reagena Ltd., Toivala, Finland) and then counted under a brightfield microscope. All experiments were performed at least 3 times and the average results were calculated.

$R N A$ extraction and RT-PCR. Total RNA was extracted from the cultured HCASMCs using TRIzol reagent according to the manufacturer's instructions (Invitrogen, Carlsbad, CA, USA). Approximately $2 \mu \mathrm{g}$ of total RNA for each sample was reverse transcribed into first-strand cDNA for RT-PCR analysis using a High-Capacity RNA-to-cDNA kit (Applied Biosystems, Foster City, CA, USA) according to the instructions of the 

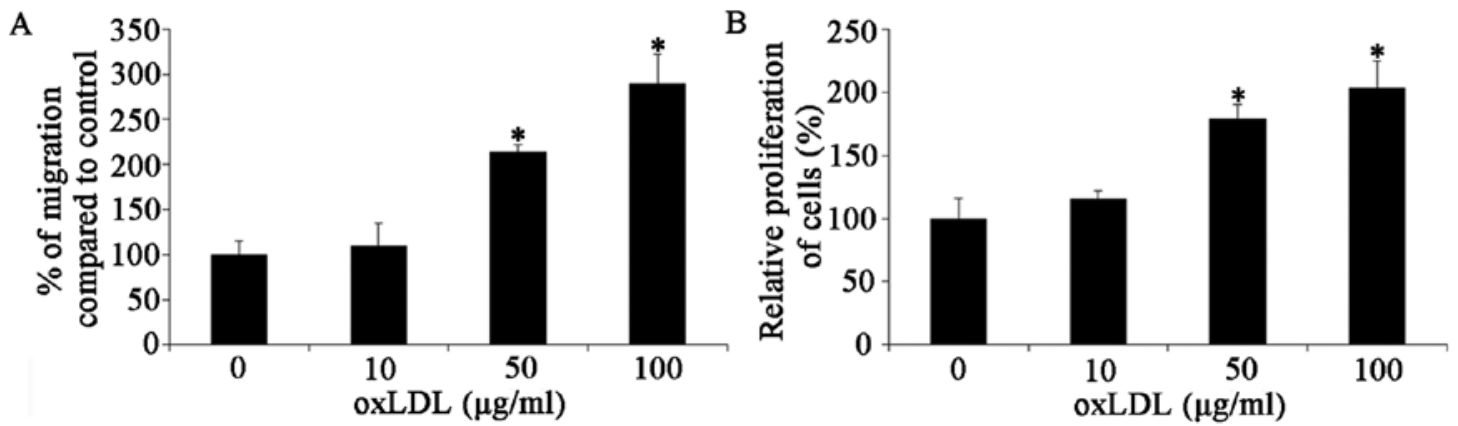

Figure 1. Effects of oxidized low-density lipoprotein (oxLDL) on human coronary artery smooth muscle cell (HCASMC) proliferation and migration. (A) Effects of oxLDL on HCASMC proliferation. Cell proliferation was detected by MTT assay. Cells were measured following treatment for $24 \mathrm{~h}$. Untreated HCASMCs were used as controls. (B) Effects of oxLDL on HCASMC migration. Cell migration was detected following treatment with oxLDL for $24 \mathrm{~h}$. Untreated HCASMCs were used as controls. All experiments were repeated at least 3 times. Data are the means \pm SEM $\left(n=5\right.$; Dunnett's test, ${ }^{*} \mathrm{P}<0.05$ compared with controls).

manufacturer. The primer sequences used for RT-PCR were as follows: OPN sense, 5'-CCAGCACAAGCAGACGTT-3' and antisense, 5'-TCAGTCCATAAGCCAAGCTATAAC-3'; MMP-9 sense, 5'-CACTGTCCACCCCTCAGAGC-3' and antisense, 5'-GCCACTTGTCGGCGATAAGC-3'; $\beta$-actin sense, 5'-CAT CCGTAAAGACCTCTATGCCAAC-3' and antisense, 5'-ATGG AGCCACCGATCCACA-3'. The primers were all synthesized by Sangon Biotech Co., Ltd. (Shanghai, China). The cycling conditions were as follows: polymerase activation for $15 \mathrm{sec}$ at $95^{\circ} \mathrm{C} ; 40$ cycles of amplification at $95^{\circ} \mathrm{C}$ for $15 \mathrm{sec}$ and $60^{\circ} \mathrm{C}$ for $60 \mathrm{sec}$. A cDNA fragment of $\beta$-actin was amplified as the control. Relative gene expression was calculated using the $2^{-\Delta \Delta c t}$ method, as previously described (29).

Western blot analysis. The total protein of HCASMCs was extracted using RIPA buffer (Beyotime, Nantong, China) according to the manufacturer's instructions. Protein concentration was measured using a BCA protein assay reagent kit (Pierce Chemical Co., Rockford, IL, USA). The proteins (40 $\mu \mathrm{g} /$ lane) were subjected to $15 \%$ SDS-PAGE followed by western blot analysis. Mouse monoclonal antibodies were used as the primary antibody and HRP-conjugated goat anti-mouse IgG was used as the secondary antibody. Finally, the blots were visualized using enhanced chemiluminescence reagents. All experiments were repeated at least 3 times.

siRNA. siRNAs targeting human OPN and MMP-9 were designed with a siRNA selection program available online at http://jura.wi.mit.edu/siRNAext/ and were chemically synthesized by Genetimes Technology (Shanghai, China). The nucleotide sequence for OPN was 5'-AAGCAGCUUUA CAACAAAUACCC-3' and 5'-AACATCACCTATTGGATC CAAACTAC-3' for MMP-9. A non-specific siRNA duplex containing the same nucleotides, but in an irregular sequence (scrambled) served as the control. The cells were transfected with siRNA (siOPN or siMMP-9) or control siRNA (siMock) using Lipofectamine 2000 (Invitrogen) according to the manufacturer's instructions. The cells were analyzed 2 days after transfection.

Statistical analysis. All data are expressed as the means \pm standard error of the mean (SEM). The differences between groups were compared by Dunnett's test subsequent to ANOVA. Differences were considered statistically significant with a value of $\mathrm{P}<0.05$. All experiments were repeated at least 3 times.

\section{Results}

oxLDL accelerates the proliferation and migration of HCASMCs. oxLDL-stimulated HCASMC responses were investigated. The effects of oxLDL on cell proliferation were determined by MTT assay. The results, shown in Fig. 1A, indicated that oxLDL at concentrations of 10, 50 and $100 \mu \mathrm{g} / \mathrm{ml}$ markedly accelerated HCASMC proliferation $(\mathrm{P}<0.05)$. oxLDL promoted the proliferation of the HCASMCs in a dose-dependent manner. We then determined the effects of oxLDL on cell migration by cell migration assay, as described in Materials and methods. Treatment of the HCASMCs with 10,50 and $100 \mu \mathrm{g} / \mathrm{ml}$ oxLDL significantly increased the migration ability of the cells to $109 \pm 26.0 \%$ at $10 \mu \mathrm{g} / \mathrm{ml}, 213 \pm 8.9 \%$ at $50 \mu \mathrm{g} / \mathrm{ml}(\mathrm{P}<0.05)$ and $289 \pm 33.1 \%$ at $100 \mu \mathrm{g} / \mathrm{ml}(\mathrm{P}<0.05)$ (Fig. 1B). These results demonstrate that oxLDL accelerates the proliferation and migration of HCASMCs; however, the exact mechanisms involved remain unelucidated. Thus, further studies are required to determine the mechanisms of action of oxLDL.

Effects of oxLDL on OPN expression. There is evidence to suggest that OPN plays an important role in the development of atherosclerotic lesions. Moreover, oxLDL increases the expression levels of OPN (11). Thus, in this study, we first detected the expression of OPN in HCASMCs following treatment with oxLDL. We used western blot analysis and RT-PCR to determine the expression of OPN in the HCASMCs. OPN mRNA expression was determined by RT-PCR following $24 \mathrm{~h}$ of incubation with oxLDL. The results revealed that oxLDL (10 to $100 \mu \mathrm{g} / \mathrm{ml}$ ) significantly increased OPN mRNA expression in the HCASMCs treated with oxLDL compared with the untreated cells (Fig. 2A). Following exposure to various doses of oxLDL, OPN expression in the HCASMCs was measured by western blot analysis. The results demonstrated that ox LDL increased the expression level of OPN following treatment with oxLDL at doses of 10 to $100 \mu \mathrm{g} / \mathrm{ml}$ compared with the controls (untreated cells; Fig. 2B). 
A

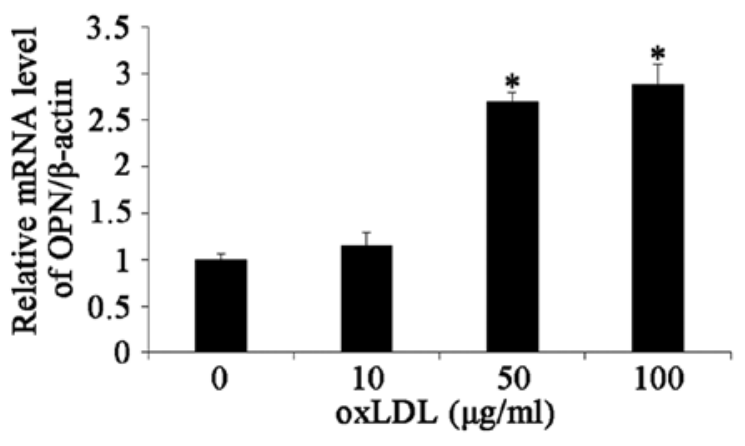

B

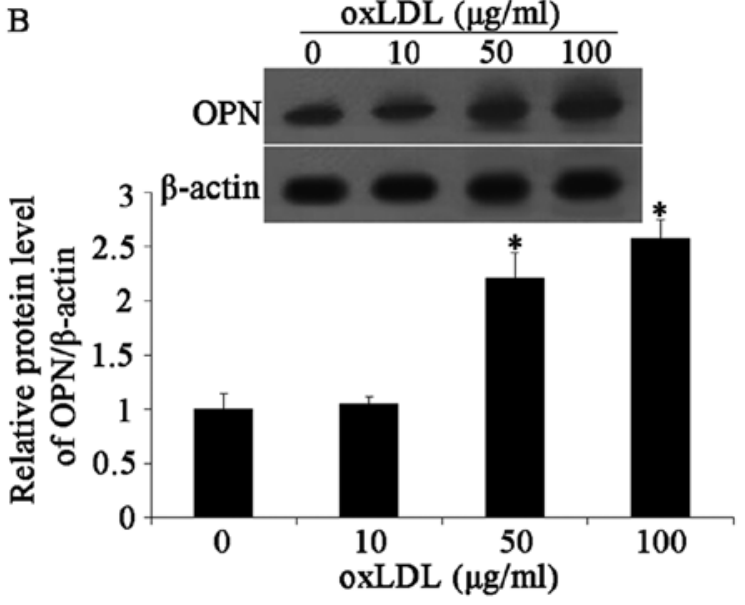

Figure 2. Oxidized low-density lipoprotein (oxLDL) increased the level of osteopontin (OPN) in human coronary artery smooth muscle cells (HCASMCs) in a dose-dependent manner. (A) OPN mRNA expression was detected by RT-PCR. Cells were treated with 10-100 $\mu \mathrm{g} / \mathrm{ml}$ oxLDL for $24 \mathrm{~h}$. OPN mRNA levels in the oxLDL-treated groups were significantly higher than those in the control group (treatment with medium only). (B) Western blot analysis of OPN expression in HCASMCs following treatment with oxLDL. OPN expression is expressed as the relative expression of OPN to $\beta$-actin expression. All experiments were repeated at least 3 times. Data are the means \pm SEM ( $n=5$; Dunnett's test, ${ }^{*} \mathrm{P}<0.05$ compared with controls).

A

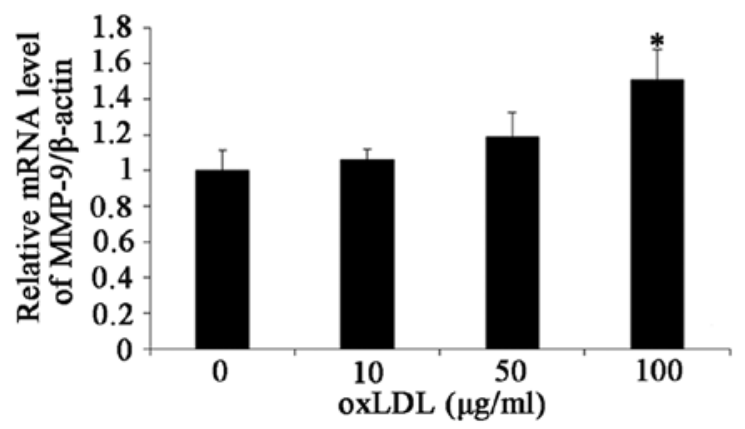

B

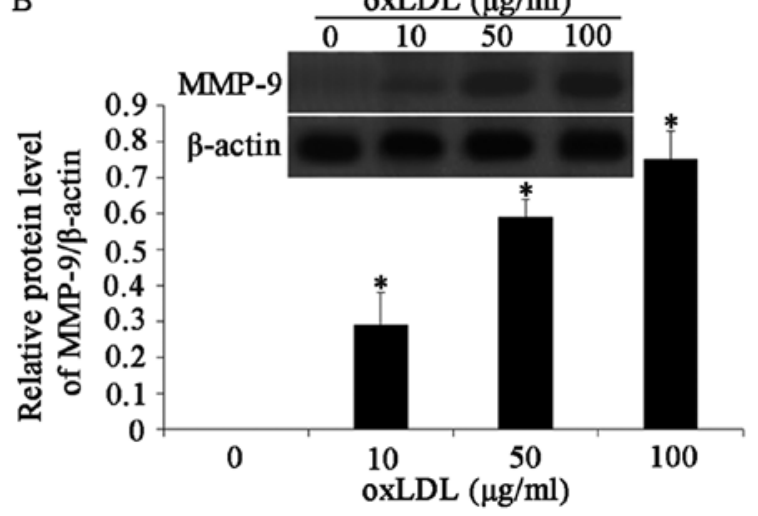

Figure 3. Effects of oxidized low-density lipoprotein (oxLDL) on matrix metaloproteinase-9 (MMP-9) expression. (A) MMP-9 mRNA level was detected by RT-PCR. Cells were treated with 10-100 $\mu \mathrm{g} / \mathrm{ml}$ oxLDL for $24 \mathrm{~h}$. OxLDL increased MMP-9 mRNA expression in HCASMCs in a dose-dependent manner. (B) Western blot analysis of MMP-9 expression in human coronary artery smooth muscle cells (HCASMCs) following treatment with oxLDL. MMP-9 expression was expressed as the relative expression of MMP-9 to $\beta$-actin expression. All experiments were repeated at least 3 times. Data are the means \pm SEM ( $=5$; Dunnett's test, ${ }^{*} \mathrm{P}<0.05$ compared with controls).

Effects of oxLDL on MMP-9 expression. A large number of studies have demonstrated that MMPs play an important role in regulating the migration and proliferation of VSMCs both in vitro and in vivo (20). There is evidence to suggest that the expression level of MMP-9 is increased during atherosclerosis in rabbits (30). The effects of oxLDL on MMP-9 expression were investigated in the HCASMCs by western blot analysis and RT-PCR. The HCASMCs were treated with oxLDL at concentrations of 10,50 and $100 \mu \mathrm{g} / \mathrm{ml}$. MMP-9 mRNA expression was significantly increased following exposure to oxLDL in a dose-dependent manner (Fig. 3A). MMP-9 protein expression was then determined by western blot analysis. As shown in Fig. 3B, the MMP-9 protein level was increased in response to oxLDL at a concentration of 10 to $100 \mu \mathrm{g} / \mathrm{ml}(\mathrm{P}<0.05)$.

OxLDL-upregulated MMP-9 expression is dependent on $O P N$ expression through the $\alpha v \beta 3$ integrin pathway. There is evidence to suggest that the treatment of chondrosarcoma or melanoma cells with OPN induces the expression of
MMP-9 (19,31). In this study, we therefore hypothesized that OPN may be involved in the oxLDL-induced upregulation of MMP-9. Hence, a siRNA experiment was performed on the HCASMCs. The OPN mRNA and protein expression levels in the siOPN-transfected group were markedly lower than those in the siMock-transfected group. The mRNA and protein expression of MMP-9 was not altered in the siOPN-transfected cells following treatment with oxLDL (Fig. 4A and B). These results indicated that the oxLDL-induced increased expression of MMP-9 was partially dependent on OPN protein expression.

We then determined the proliferation and migration ability of the HCASMCs. The results revealed that cell proliferation and migration were markedly reduced following treatment with OPN siRNA. The silencing of OPN partially reduced the increased proliferation and migration resulting from treatment with oxLDL (Fig. 4C). A previous study suggested that OPN affects cell migration through $\alpha v \beta 3$ integrin signaling (32). Thus, we hypothesized that the $\alpha v \beta 3$ integrin signaling pathway may be involved in the biological actions of 
A

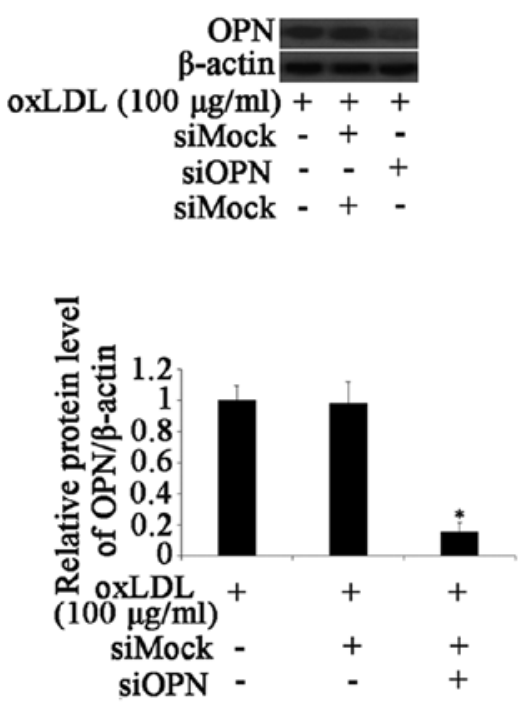

D
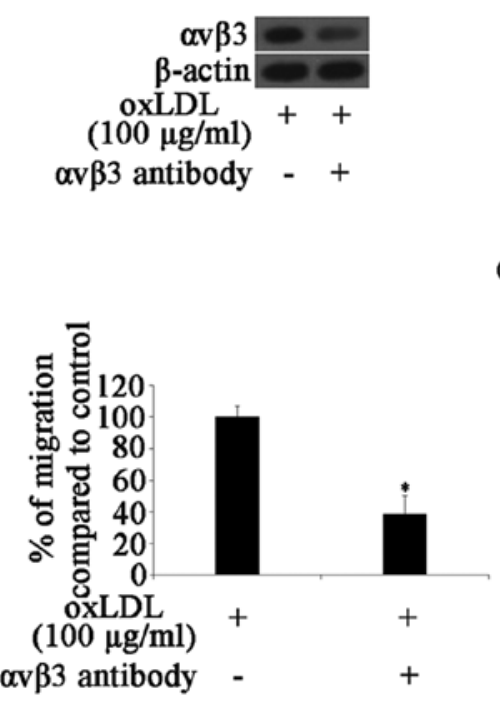

B
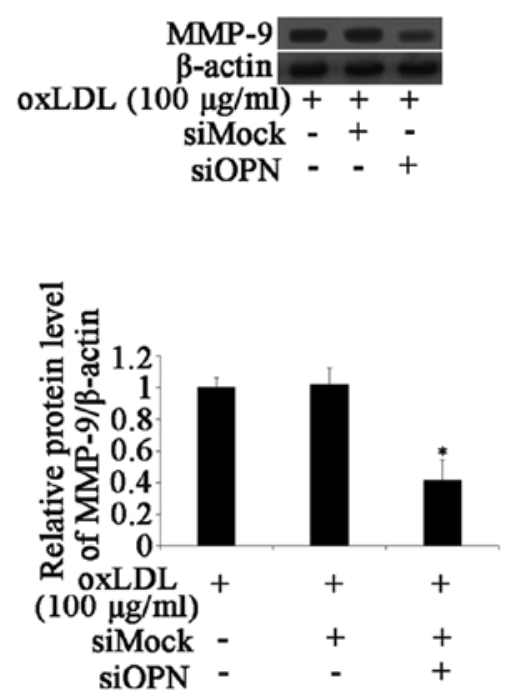

E $\vec{D}$

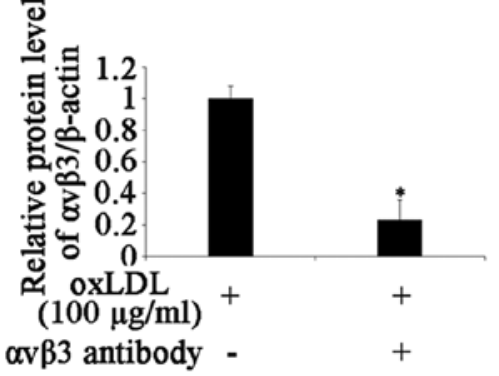

$\alpha v \beta 3$ antibody -

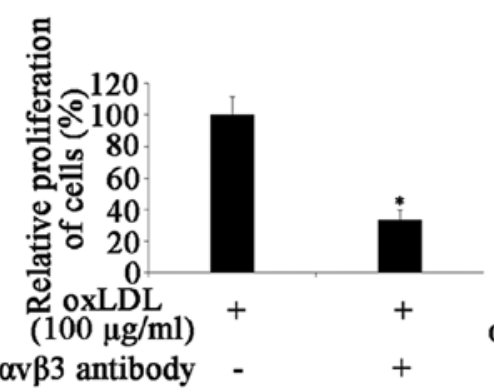

$\mathrm{C}$
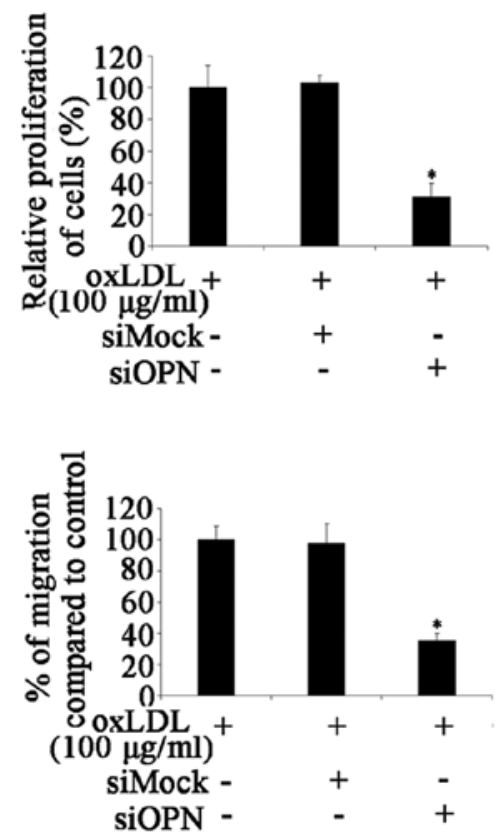

$\mathbf{F}$
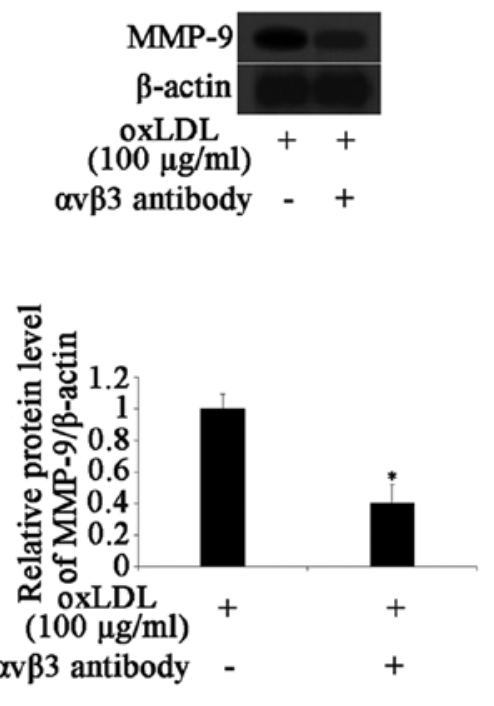

Figure 4. Effects of osteopontin (OPN) on matrix metaloproteinase-9 (MMP-9) expression and cell proliferation and migration of human coronary artery smooth muscle cells (HCASMCs) and siOPN cells following treatment with $100 \mu \mathrm{g} / \mathrm{ml}$ oxidized low-density lipoprotein (oxLDL). (A) The expression of OPN in HCASMCs and siOPN cells following treatment with oxLDL was measured by western blot analysis. OPN expression levels were normalized to $\beta$-actin. (B) The expression of MMP-9 in HCASMCs and siOPN cells following treatment with oxLDL was detected by western blot analysis. MMP-9 expression levels were normalized to $\beta$-actin. (C) The proliferation and migration of HCASMCs and siOPN cells following treatment with oxLDL. (D) The expression of $\alpha v \beta 3$ integrin in HCASMCs following treatment with $\alpha v \beta 3$ integrin monoclonal antibody was measured by western blot analysis. $\alpha v \beta 3$ integrin expression level was normalized to $\beta$-actin. (E) The proliferation and migration of HCASMCs following treatment with $\alpha v \beta 3$ antibody. (F) The expression of MMP-9 in HCASMCs following treatment with $\alpha v \beta 3$ antibody was measured by western blot analysis. MMP-9 expression level was normalized to $\beta$-actin. All experiments were repeated at least 3 times. Data are the means \pm SEM ( $n=5$; Dunnett's test, ${ }^{*} \mathrm{P}<0.05$ compared with controls).

HCASMCs. Pre-treatment of the cells for $30 \mathrm{~min}$ with $20 \mu \mathrm{g} / \mathrm{ml}$ anti- $\alpha v \beta 3$ integrin monoclonal antibody markedly reduced the expression of $\alpha v \beta 3$ integrin (Fig. 4D). We then determined the effects of anti- $\alpha v \beta 3$ integrin monoclonal antibody on the proliferation and migration ability of the HCASMCs. The results revealed that the antibody inhibited oxLDL-induced cell proliferation and migration (Fig. 4E). At the same time, the expression of MMP-9 was detected. The results revealed that pre-treatment of the cells with anti- $\alpha v \beta 3$ antibody decreased oxLDL-induced MMP-9 expression (Fig. 4F). These results indicated that oxLDL increased the proliferation and migration of the HCASMCs through the upregulation of OPN and MMP-9, and that MMP-9 expression is partly dependent on OPN expression through the $\alpha v \beta 3$ integrin pathway. 
A

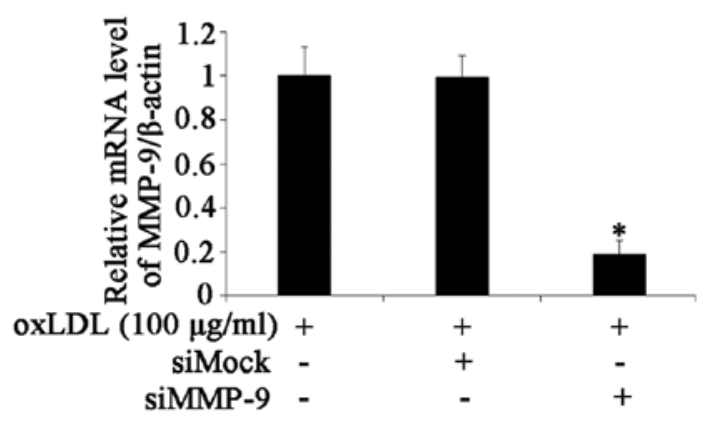

B

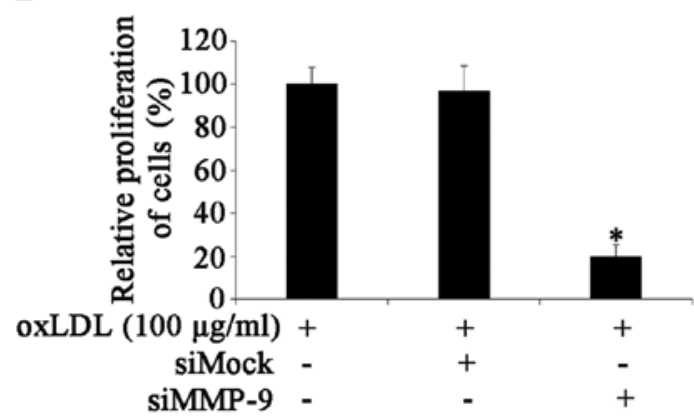

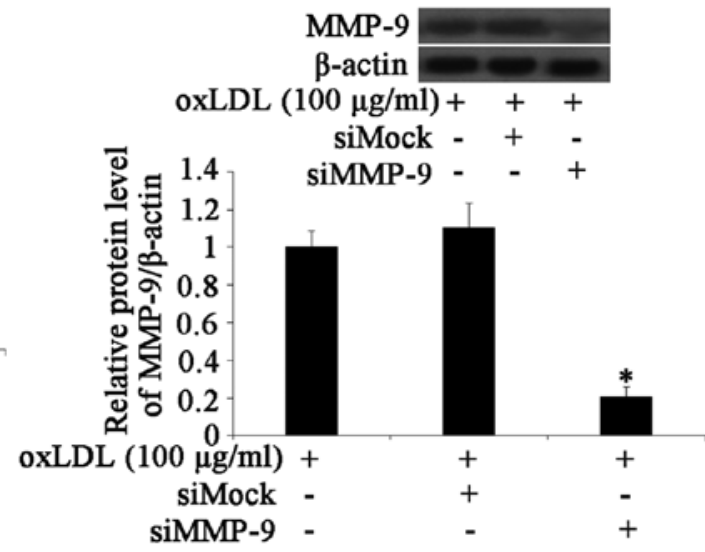

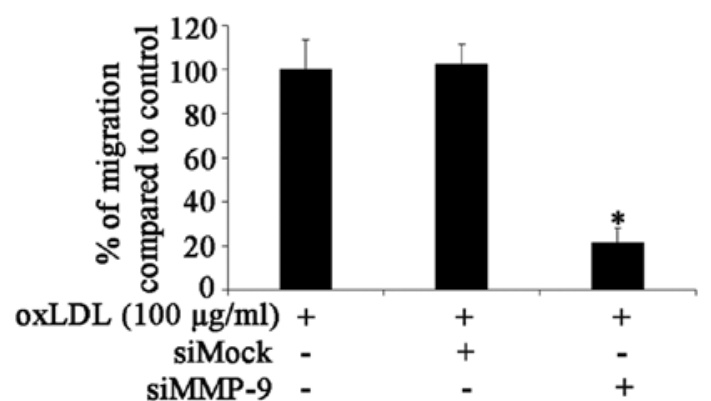

Figure 5. Effects of matrix metaloproteinase-9 (MMP-9) on human coronary artery smooth muscle cell (HCASMC) proliferation and migration following treatment with $100 \mu \mathrm{g} / \mathrm{ml}$ oxidized low-density lipoprotein (oxLDL) . (A) The expression of MMP-9 in HCASMCs and siMMP-9 cells following treatment with oxLDL was measured by western blot analysis. MMP-9 expression level was normalized to $\beta$-actin. (B) The proliferation and migration of HCASMCs and siMMP-9 cells following treatment with oxLDL. All experiments were repeated at least 3 times. Data are the means \pm SEM ( $=5$; Dunnett's test, ${ }^{*} \mathrm{P}<0.05$ compared with controls).

Effects of siMMP-9 on HCASMCs. To determine the effects of MMP-9 on the behavior of HCASMCs, a siRNA experiment was performed. MMP-9 mRNA and protein expression levels in the siMMP-9-transfected group were markedly lower than those in the siMock-transfected group (Fig. 5A). Cell proliferation and migration were markedly reduced after the cells were treated with MMP-9 siRNA. The silencing of MMP-9 markedly reduced the effects of oxLDL on HCASMC proliferation and migration (Fig. 5B). These results indicate that MMP-9 plays an important role in the oxLDL-induced proliferation and migration of HCASMCs.

\section{Discussion}

Elucidating the mechanisms that result in the initiation and development of atherosclerosis is crucial for identifying strategies to inhibit disease progression before it leads to clinical consequences (33). A large number of studies have indicated that many cell types, such as endothelial cells, lymphocytes, macrophages and SMCs are associated with the formation of atherosclerotic lesions (34). There is evidence to suggest that SMC migration and proliferation play a major role in the formation of fibroproliferative plaques (35). A predominant feature of atherogenesis is SMC migration into the intima of the arterial wall where they synthesize the ECM, together with the accumulation of cholesterol and proliferation by macrophages, leading to the progression of atherosclerotic plaque (36). SMC proliferation is a critical mechanism for the formation of plaque and fibrous caps; these cells are also involved in endothelial cell injury, necrotic core formation and plaque rupture $(34,37)$.

A number of factors affect SMC proliferation and migration, including hypertension, increased plasma cholesterol levels and oxidative stress. oxLDL, present in atherosclerotic lesions, is a well-established risk factor for atherosclerosis that induces changes in gene expression (growth factors, cytokines and adhesion molecules), lipoprotein metabolism, cell migration, proliferation and apoptosis (38). An important finding of this study was that OPN contributes to the oxLDL-induced migration and proliferation of HCASMCs. In this study, we determined whether the expression of OPN is upregulated when the HCASMCs were exposed to oxLDL. The results demonstrated that oxLDL stimulated the expression of OPN; there was a positive correlation between the OPN expression level and HCASMC proliferation and migration.

There is evidence indicating that oxLDL upregulates the expression of OPN (11). Moreover, high levels of OPN are associated with the upregulation of MMP-9 levels (19). We therefore, hypothesized that OPN may be involved in the oxLDL-induced upregulation of MMP-9. To further confirm the role of OPN and to clarify the mechanisms underlying the oxLDL-induced increase in cell proliferation and migration, MMP-9 expression was detected in the HCASMCs. The results revealed that treatment with oxLDL induced a significant increase in MMP-9 expression, which was dependent on OPN expression levels. Previous studies have suggested that OPN upregulates the expression of MMP-9 through the $\alpha v \beta 3$ integrin pathway (19). Another finding of this study was that oxLDL activates $\alpha v \beta 3$ 
integrin in HCASMCs. We found that the level of $\alpha v \beta 3$ integrin was markedly reduced following the treatment of the cells with antibody against $\alpha v \beta 3$. Moreover, MMP-9 expression, as well as the cell proliferation and migration, were also reduced. To further confirm the role of MMP-9 in HCASMC proliferation and migration, a siRNA experiment for MMP-9 was performed. The results demonstrated that cell proliferation and migration were markedly reduced following the treatment of the cells with MMP-9 siRNA. These results indicate that oxLDL increases the proliferation and migration of HCASMCs through the upregulation of OPN and MMP-9, and that MMP-9 expression is partly dependent on OPN expression through the $\alpha v \beta 3$ integrin pathway.

In conclusion, our data demonstrate that oxLDL promotes the proliferation and migration of HCASMCs through the upregulation of the expression of OPN and MMP-9. Furthermore, our results suggest that the effects of oxLDL on the expression of MMP-9 are dependent on the expression of OPN through the $\alpha v \beta 3$ integrin pathway. The data presented in our study provide new insight into the mechanisms through which oxLDL affects HCASMC proliferation and migration.

\section{References}

1. Wang XH, Wang F, You SJ, et al: Dysregulation of cystathionine $\gamma$-lyase (CSE)/hydrogen sulfide pathway contributes to ox-LDL-induced inflammation in macrophage. Cell Signal 25: 2255-2262, 2013.

2. Glass CK and Witztum JL: Atherosclerosis: the road ahead. Cell 104: 503-516, 2001.

3. González-Navarro H, Abu Nabah YN, Vinué Á, et al: p19(ARF) deficiency reduces macrophage and vascular smooth muscle cell apoptosis and aggravates atherosclerosis. J AM Coll Cardiol 55: 2258-2268, 2010.

4. Gerthoffer WT: Mechanisms of vascular smooth muscle cell migration. Circ Res 100: 607-621, 2007.

5. Murry CE, Gipaya CT, Bartosek T, Benditt EP and Schwartz SM: Monoclonality of smooth muscle cells in human atherosclerosis. Am J Pathol 151: 697-705, 1997.

6. Johnson JL, Dwivedi A, Somerville M, George SJ and Newby AC: Matrix metalloproteinase (MMP)-3 activates MMP-9 mediated vascular smooth muscle cell migration and neointima formation in mice. Arterioscl Thromb Vas 31: e35-e 44, 2011.

7. Li LX, Zhang XF, Bai X and Tong Q: SDF-1 promotes ox-LDL induced vascular smooth muscle cell proliferation. Cell Biol Int 37: 988-994, 2013.

8. Ross R: The pathogenesis of atherosclerosis - an update. New Engl J Med 314: 488-500, 1986.

9. Clarke MC, Figg N, Maguire JJ, et al: Apoptosis of vascular smooth muscle cells induces features of plaque vulnerability in atherosclerosis. Nat Med 12: 1075-1080, 2006.

10. Bennett MR and Boyle JJ: Apoptosis of vascular smooth muscle cells in atherosclerosis. Atherosclerosis 138: 3-9, 1998.

11. Mazière C, Gomila C and Mazière JC: Oxidized low-density lipoprotein increases osteopontin expression by generation of oxidative stress. Free Radical Bio Med 48: 1382-1387, 2010.

12. Cho HJ, Cho HJ and Kim HS: Osteopontin: a multifunctional protein at the crossroads of inflammation, atherosclerosis, and vascular calcification. Curr Atheroscler Rep 11: 206-213, 2009.

13. Giachelli CM, Lombardi D, Johnson RJ, Murry CE and Almeida M: Evidence for a role of osteopontin in macrophage infiltration in response to pathological stimuli in vivo. Am J Pathol 152: 353-358, 1998.

14. Weber GF, Zawaideh S, Hikita S, Kumar VA, Cantor H and Ashkar S: Phosphorylation-dependent interaction of osteopontin with its receptors regulates macrophage migration and activation. J Leukocyte Biol 72: 752-761, 2002.

15. Koh A, Da Silva AP, Bansal AK, et al: Role of osteopontin in neutrophil function. Immunology 122: 466-475, 2007.

16. Ikeda T, Shirasawa T, Esaki Y, Yoshiki S and Hirokawa K: Osteopontin mRNA is expressed by smooth muscle-derived foam cells in human atherosclerotic lesions of the aorta. J Clin Invest 92: 2814-2820, 1993.
17. Zohar R, Suzuki N, Suzuki K, et al: Intracellular osteopontin is an integral component of the CD44-ERM complex involved in cell migration. J Cell Physiol 184: 118-130, 2000.

18. Shannon KE, Keene JL, Settle SL, et al: Anti-metastatic properties of RGD-peptidomimetic agents S137 and S247. Clin Exp Metastasis 21: 129-138, 2004

19. Chen YJ, Wei YY, Chen HT, et al: Osteopontin increases migration and MMP-9 up-regulation via $\alpha v \beta 3$ integrin, FAK, $\mathrm{ERK}$, and NF- $\mathrm{BB}$-dependent pathway in human chondrosarcoma cells. J Cell Physiol 221: 98-108, 2009.

20. Newby AC: Matrix metalloproteinases regulate migration, proliferation, and death of vascular smooth muscle cells by degrading matrix and non-matrix substrates. Cardiovasc Res 69: 614-624, 2006.

21. Johnson C and Galis ZS: Matrix metalloproteinase-2 and -9 differentially regulate smooth muscle cell migration and cell-mediated collagen organization. Arterioscl Thromb Vasc Biol 24: 54-60, 2004.

22. Kuzuya M, Kanda S, Sasaki T, et al: Deficiency of gelatinase a suppresses smooth muscle cell invasion and development of experimental intimal hyperplasia. Circulation 108: 1375-1381, 2003.

23. Johnson JL, George SJ, Newby AC and Jackson CL: Divergent effects of matrix metalloproteinases $3,7,9$, and 12 on atherosclerotic plaque stability in mouse brachiocephalic arteries. Proc Natl Acad Sci USA 102: 15575-15580, 2005.

24. Belcher J, Egan JO, Bridgman G, Baker R and Flack JM: A micro-enzymatic method to measure cholesterol and triglyceride in lipoprotein subfractions separated by density gradient ultracentrifugation from 200 microliters of plasma or serum. J Lipid Res 32: 359-370, 1991

25. Feng X, Zhang Y, Xu R, et al: Lipopolysaccharide up-regulates the expression of Fcalpha/mu receptor and promotes the binding of oxidized low-density lipoprotein and its IgM antibody complex to activated human macrophages. Atherosclerosis 208 : 396-405, 2010.

26. Lowry OH, Rosebrough NJ, Farr AL and Randall RJ: Protein measurement with the Folin phenol reagent. J biol Chem 193: 265-275, 1951.

27. Shyu KG, Chen SC, Wang BW, Cheng WP and Hung HF: Mechanism of the inhibitory effect of atorvastatin on leptin expression induced by angiotensin II in cultured human coronary artery smooth muscle cells. Clin Sci (Lond) 122: 33-42, 2012.

28. Pellet-Many C, Frankel P, Evans IM, Herzog B, JünemannRamirez $M$ and Zachary IC: Neuropilin-1 mediates PDGF stimulation of vascular smooth muscle cell migration and signalling via p130Cas. Biochem J 435: 609-618, 2011.

29. Livak KJ and Schmittgen TD: Analysis of relative gene expression data using real-time quantitative PCR and the 2(-Delta Delta C(T)) Method. Methods 25: 402-408, 2001

30. Aikawa M, Rabkin E, Okada Y, et al: Lipid lowering by diet reduces matrix metalloproteinase activity and increases collagen content of rabbit atheroma: a potential mechanism of lesion stabilization. Circulation 97: 2433-2444, 1998.

31. Rangaswami H and Kundu GC: Osteopontin stimulates melanoma growth and lung metastasis through NIK/MEKK1-dependent MMP-9 activation pathways. Oncol Rep 18: 909-915, 2007.

32. Wai PY and Kuo PC: Osteopontin: regulation in tumor metastasis. Cancer Metastasis Rev 27: 103-118, 2008.

33. Doran AC, Meller N and McNamara CA: Role of smooth muscle cells in the initiation and early progression of atherosclerosis. Arterioscler Thromb Vasc Biol 28: 812-819, 2008.

34. Lusis AJ: Atherosclerosis. Nature 407: 233-241, 2000.

35. Augé N, Maupas-Schwalm F, Elbaz M, et al: Role for matrix metalloproteinase-2 in oxidized low-density lipoprotein-induced activation of the sphingomyelin/ceramide pathway and smooth muscle cell proliferation. Circulation 110: 571-578, 2004.

36. Ricciarelli R, Zingg JM and Azzi A: Vitamin E reduces the uptake of oxidized LDL by inhibiting CD36 scavenger receptor expression in cultured aortic smooth muscle cells. Circulation 102: 82-87, 2000

37. Auge N, Garcia V, Maupas-Schwalm F, Levade T, Salvayre R and Negre-Salvayre A: Oxidized LDL-induced smooth muscle cell proliferation involves the EGF receptor/PI-3 kinase/Akt and the sphingolipid signaling pathways. Arterioscl Thromb Vasc Biol 22: 1990-1995, 2002.

38. Witztum JL and Steinberg D: The oxidative modification hypothesis of atherosclerosis: does it hold for humans? Trends Cardiovas Med 11: 93-102, 2001. 